\title{
ASPECTOS PSICOSOCIALES Y CONDICIONES EDUCATIVAS DE DOCENTES DE LENGUA EN FORMACIÓN DURANTE EL CONFINAMIENTO POR LA PANDEMIA DE COVID-19
}

\author{
fl Pre-Service Teachers' Psychosocial Aspects and Educational Conditions \\ DURING THE COVID-19 PANDEMIC LOCKDOWN
}

ASPECTS PSYCHOSOCIAUX ET CONDITIONS ÉDUCATIVES CHEZ DES ENSEIGNANTS DE LANGUES EN FORMATION PENDANT LE CONFINEMENT PAR LA PANDÉMIE DE COVID-19

\author{
Jacqueline García Botero \\ Docente de Inglés, Universidad del \\ Quindío, Armenia, Colombia. \\ igarciab@uniquindio.edu.co \\ https://orcid. \\ org/0000-0002-9850-7947

\section{Gustavo García Botero \\ Docente en tecnologías educativas e interdisciplinariedad, Vrije Universiteit Brussel, Ixelle, Bélgica. gustavo.garcia.botero@vub.be https://orcid.} \\ org/0000-0003-1606-9486
}

\author{
Margarita Alexandra Botero \\ Restrepo \\ Profesora asociada de Francés, \\ Universidad del Quindío, Armenia, \\ Colombia. \\ mabotero@uniquindio.edu.co \\ https://orcid. \\ org/0000-0001-7534-3874
}

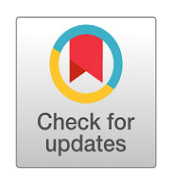

\begin{abstract}
RESUMEN
Dada la contingencia sanitaria mundial a causa de la covid-19, los escenarios educativos en todo el mundo se convirtieron en "laboratorios investigativos", en los cuales cobraron gran importancia los procesos de enseñanza-aprendizaje mediados por la tecnología. Por esto, la presente investigación tuvo como objetivo principal identificar los aspectos psicosociales y las condiciones educativas que influyeron en la satisfacción con el aprendizaje de 423 estudiantes pertenecientes a un programa de Licenciatura en Lenguas Modernas con énfasis en Inglés y Francés de una Universidad en Colombia que empleó la metodología de presencialidad asistida por la tecnología (PAT). Para esto, se implementaron cuestionarios abiertos, grupos focales y observaciones participativas, los cuales fueron analizados siguiendo las etapas del análisis de datos cualitativos planteadas por Miles et al. (2014) y los procesos de codificación propuestos por McCracken (1988). Se concluyó que las categorías determinantes que inciden en la satisfacción del estudiante son el apoyo del profesor y las condiciones estructurales. Estas dos categorías tienen un impacto en otros aspectos psicosociales que se convierten en un desafío para la metodología PAT. Los resultados señalan la necesidad de una reflexión pedagógica que ayude a unificar criterios para una implementación apropiada de esta metodología.
\end{abstract}

Palabras clave: aprendizaje en línea; aspectos psicosociales; COVID-19; enseñanza de lengua extranjera; presencialidad asistida por tecnología; TIC; condiciones educativas.

\section{Abstract}

Face to the global health contingency due to CoviD-19, educational settings around the world have become a "research laboratory" in which technology-mediated

Recibido: 2021-09-09 / Aceptado: 2021-05-28 / Publicado: 2021-09-11

https://doi.org/10.17533/udea.ikala.v26n3a05

Edición especial sobre The Role of Technology in Language Teaching and Learning amid the Crisis Generated by the COVID-19 Pandemic.

Editores: Marta González-Lloret, University of Hawai'i at Mānoa, Ee.UU.; Laia Canals, Universitat Oberta de Catalunya, España; Jorge Pineda, Universidad de Antioquia, Colombia.

(C) 2021 Universidad de Antioquia. Este artículo se difunde en acceso abierto, según lo establecido en la Licencia Internacional 4.0 de Creative Commons BY-NC-SA. 
teaching-learning processes have gained increasing importance. This research had as a main goal to identify the psychosocial aspects and educational conditions that influenced the degree of learning satisfaction of 423 students enrolled in a BA in Modern Languages (English and French) at a university in Colombia that employed technology-mediated 'face-to-face' learning (PAT, as per its Spanish acronym). Data were collected through open-ended questionnaires, focus groups and participant observations, and were analyzed following the qualitative data analysis stages proposed by Miles et al., and McCracken's (1988) coding processes. Findings show that students' learning satisfaction is highly influenced by teacher's support and structural conditions. These two categories have an impact on other psychosocial conditions that become a challenge for the PAT technology. The results show the need for a pedagogical reflection that helps instructors unify criteria for an appropriate implementation of this methodology.

Keywords: E-learning; psychosocial aspects; Covid-19; foreign language teaching; technology-mediated face-to-face learning; ICT; educational conditions.

\section{RÉSUMÉ}

Compte tenu de la contingence sanitaire mondiale de la covid-19, les milieux éducatifs du monde entier sont devenus un «laboratoire de recherche » dans lequel les processus d'enseignement-apprentissage à médiation technologique ont gagné plus d'importance. Cette recherche vise à identifier les aspects psychosociaux et les conditions éducatives qui ont influencé la satisfaction dans l'apprentissage de 423 étudiants dans une université en Colombie qui utilise l'enseignement presentielle médiée par la technologie (PAT). Pour ça faire, des questionnaires ouverts, des groupes focaux et des observations participatives ont été mis en place et ont été analysés suivant les étapes d'analyse des données qualitatives proposées par Miles et al., et les processus d'encodage proposés par McCracken (1998). Les résultats montrent que les catégories déterminantes qui affectent la satisfaction des élèves sont le soutien des enseignants et les conditions structurelles. Ces deux catégories ont un impact sur d'autres aspects psychosociaux qui deviennent un défi pour la métodologie PAT. Les résultats indiquent aussi la nécessité d'une réflexion pédagogique pour aider les enseignants à unifier les critères pour une mise en œuvre appropriée de cette méthodologie.

Mots-clés : apprentissage en ligne; aspects psychosociaux ; COVID-19; enseignement des langues étrangères; méthodologie en présentiel assistée par la technologie ; TIC ; conditions éducatives. 


\section{Introducción}

Sin duda, la emergencia sanitaria ocasionada por la COviD-19 "ha dado un giro a la educación de lenguas extranjeras al igual que lo ha hecho en todos los ámbitos de la educación" (Pardo, 2020, p. 5), obligando tanto a profesores como a estudiantes a adaptarse al nuevo entorno académico mediado por la tecnología. No obstante, esta irrupción de la tecnología en el contexto de enseñanza y aprendizaje de lenguas no es algo nuevo; ya lo advertían Kukulska-Hulme (2009) y Mac Callum et al. (2014), quienes resaltan el interés que ha ganado la enseñanza de lenguas por medio de tecnologías móviles. El contexto mundial, nacional y local que ha generado la covid-19, le ha otorgado a la mediación tecnológica un rol protagónico y pone en evidencia la necesidad de repensar dicho rol, de reconstruirlo mediante un "desaprender", en términos piagetianos (Tünnermann, 2011), para reaprender y resignificar el lugar que se le debe otorgar a dichos recursos tecnológicos.

En Colombia, desde que se decretó la emergencia sanitaria en el país el 12 de marzo de 2020, el Ministerio de Educación Nacional (MEN) ha generado diferentes lineamientos con el fin de facilitar el proceso pedagógico en casa y dar continuidad a la oferta educativa en todos los niveles educativos. Se insistió en hacer uso de herramientas como e-Learning, portales de conocimiento, redes sociales y plataformas colaborativas, para adelantar los procesos de capacitación y formación que sean inaplazables" (Colombia, Presidencia de la República, 2020). Así, las instituciones de educación superior, bajo el concepto de autonomía universitaria, han planteado diferentes estrategias para afrontar este gran desafío y seguir ofreciendo su servicio educativo, ya sea de manera remota o considerando una modalidad semipresencial.

Para el caso concreto de la Universidad del Quindío, y específicamente el programa de Lenguas Modernas, se desarrollaron sus actividades académicas de manera virtual, siguiendo la estrategia metodológica de presencialidad asistida por la tecnología (PAT). Ya que esta estrategia representa una novedad en las practicas tradicionales de la universidad, y en gran parte del sistema educativo de educacion superior colombiano, el presente estudio plantea la siguiente pregunta de investigación: ¿cuáles aspectos psicosociales y condiciones educativas influyen en la satisfacción de estudiantes del programa de Licenciatura en Lenguas Modernas de la Universidad del Quindío durante su experiencia de aprendizaje bajo la estrategia metodológica PAT? Dichos aspectos psicosociales fueron abordados teniendo en cuenta el cuestionario DELES (Distance Education Learning Environment Survey) propuestos por Walker y Fraser $(2005)^{1}$ y adaptado a la nueva realidad educativa; además, se incluyeron otros aspectos psicosociales como la salud física y mental.

\section{Marco teórico}

Antes de ahondar en los aspectos psicosociales y las condiciones educativas analizados desde este estudio, es importante analizar y aclarar cuáles son las metodologías avaladas por el MEN para otorgar registro calificado o renovación de registro a programas académicos, ya que cada modalidad deriva unas características específicas para el tipo de estudiante colombiano que escoge entre una y otra. Dichas metodologías han sido clasificadas bajo la modalidad "presencial", "distancia" y "virtual”.

En lo que respecta a la "educación presencial", esta es "un acto comunicativo donde un profesor imparte clases a sus alumnos, en un mismo lugar y tiempo" (Romero et al., 2014, p. 3). Dicho espacio y tiempo son sincrónicos, determinados y establecidos por las decisiones estipuladas por el MEN.

Teniendo en cuenta la democratización de la educación como un derecho fundamental y que los espacios presenciales no responden siempre a las

1 Cuestionario DELES (Distance Education Learning Environment Survey) DELES - Copyright (C2020 by Scott L. Walker. All rights reserved in all media. Published by Mind Garden, Inc., www.mindgarden.com. 
realidades y dinámicas económicas, surge como alternativa a los problemas de calidad y cobertura la modalidad de "educación a distancia". Esta se entiende como

[...] una modalidad educativa que comprende una situación formal de enseñanza y de aprendizaje donde profesor y estudiante, comunidad académica y actores organizacionales, se encuentran en una dimensión tiempo-espacio distinta (Asociación Colombiana de Instituciones de Educación Superior con Programas a Distancia, 2013, p. 18).

Esta mediación puede recurrir (y no de manera exclusiva) al uso de las TIC, y no excluye el encuentro docente-alumno de manera presencial.

En cuanto a la "educación virtual o en línea", el MEN la clasifica como una posibilidad (en medio de muchas otras) de la educación a distancia. Particularmente, la concibe como

[...] desarrollo de un proceso educativo en un lugar distinto al salón de clases: en el ciberespacio; en una temporalidad que puede ser síncrona o asíncrona y sin la necesidad de que los cuerpos de maestros y estudiantes estén presentes. Para ello se usan las redes telemáticas que se constituyen en su entorno principal (Colombia, MEN, 2010, p. 33).

Esta definición sitúa a las TIC en uno de los pilares educativos, y no al tiempo ni al espacio. El encuentro educativo se modifica y no se centra en el cara a cara en un mismo momento (sincronía), o en un mismo lugar (aula), sino en una nueva dinámica, que puede ser también asincrónica, en una proliferación de espacios congregados en la virtualidad.

La educación virtual o en línea, si bien ha tenido un papel importante en la educación como una de las alternativas para acceder a ella, pasó de ser una elección a ser una exclusividad en tiempos de pandemia.

El contexto nacional aporta igualmente a las nuevas estrategias y denominaciones de la enseñanza en tiempos de crisis. Más particularmente, la Universidad EAN propone la metodología PAT para continuar con el proceso educativo. Esta metodología ha sido orientada institucionalmente a través del comunicado institucional número 2 del 13 de marzo de 2020, en el que la funda como el principal mecanismo para dar continuidad a las actividades académicas establecidas en la Universidad. Así mismo, enuncia las características de esta metodología emergente y las ventajas de su implementación (Universidad EAN, 2020). PAT consiste en continuar con el proceso académico de la misma manera como se venía haciendo desde la presencialidad, pero por las medidas de confinamiento que se deben adoptar, desde la virtualidad.

La implementación de esta metodología se caracteriza por la conservación tanto del número de clases como de la intensidad horaria; el contacto directo con el docente (por medio de encuentros sincrónicos), en los mismos horarios de clase y las mismas actividades planificados previamente desde la presencialidad; procurando un ambiente activo y participativo.

\section{Aspectos psicosociales}

Hablar de "condiciones psicosociales" remite a entender al sujeto y su relación con el entorno. Leal (2005) define los aspectos psicosociales como aquellas variables presentes en los contextos sociales que tienen un impacto en el desarrollo y el bienestar psicológico del individuo.

El cambio abrupto generado por la pandemia en el funcionamiento del sistema educativo ocasionó sin duda una serie de situaciones que confluían en el rendimiento académico, y en la salud física y mental de la comunidad académica. Y es que el aprendizaje de lenguas extranjeras se ve altamente influenciado por aspectos psicosociales, entre otros, la motivación, que resulta ser una "pieza facilitadora en la adquisición de una L2 [segunda lengua]" (Fonseca, 2005); la interacción, como factor indisociable de los procesos de enseñanza y aprendizaje de lenguas (Govea y Sánchez, 2006); y la autonomía, como proceso evolutivo que potencia el reconocimiento de los estilos de aprendizaje 


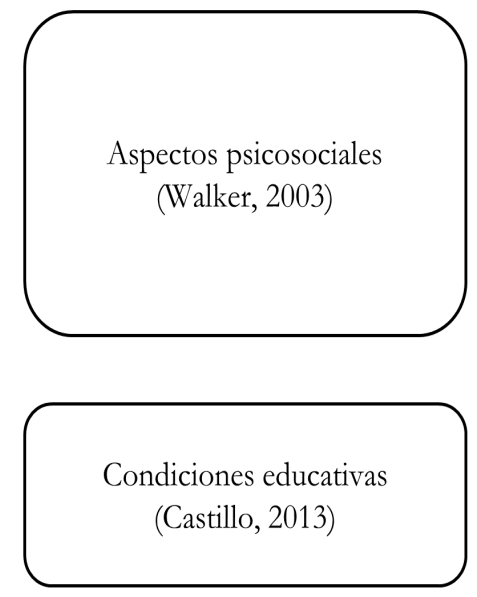

Apoyo del profesor

Aprendizaje activo

Aprendizaje autónomo

Interacción estudiantil

Aprendizaje auténtico/relevante

Salud física y mental

- Infraestructura física

- Medios educativos

Figura 1 Aspectos psicosociales y condiciones educativas en la satisfacción estudiantil en el aprendizaje mediado por tecnología digital

y el autodireccionamiento del conocimiento para lograr mejor desempeño en el aprendizaje de lenguas (Velázquez et al., 2016).

Así, desde el presente estudio se analizaron los siguientes aspectos psicosociales propuestos por Walker (2003), teniendo en cuenta la mediación tecnológica como eje central: el apoyo docente, el aprendizaje activo y autónomo, la interacción entre los estudiantes, el aprendizaje auténtico y relevante. La Figura 1 resume los aspectos psicosociales y las condiciones educativas que se tomaron en cuenta en la presente investigación.

Considerando el apoyo docente, Chaves (2017) sostiene que, cuando hay mediación tecnológica, los docentes deben procurar el uso de diferentes herramientas que mantengan activo el contacto con los estudiantes; así, el rol docente debe ser activo, procurando la comunicación bidireccional y el acompañamiento continuo (Morales, 2014). El aprendizaje activo y autónomo, por su parte, se entiende como el rol del estudiante en la construcción de su propio conocimiento; el reconocimiento de su estilo de aprendizaje, la toma de decisiones, su autorregulación.
La interacción entre los estudiantes, como aspecto psicosocial, se comprende como el conocimiento adquirido que se ve complementado con el trabajo de los otros y cómo se posibilita acrecentar las experiencias de aprendizaje a partir de la colaboración entre pares. Pérez (2009) destaca la variable "interacción” como una de las principales causales de la deserción en la modalidad a distancia, pues considera que el proceso de aprendizaje se ve acrecentado en la medida en que el clima socioafectivo sea propicio.

Finalmente, en el aprendizaje auténtico y relevante, se analizan las relaciones entre lo aprendido y el mundo exterior; en otras palabras, la existencia de un aprendizaje significativo. Sumado a lo anterior, este estudio se interesó también por analizar la salud física y mental de los estudiantes durante el confinamiento y a partir de la implementación del modelo PAT.

\section{Condiciones educativas}

Todo proceso educativo requiere de unas condiciones mínimas que les permitan a sus participantes lograr los objetivos establecidos para el desarrollo de competencias tanto individuales como colectivas. 
En el contexto de educación superior, dichas condiciones están reglamentadas por dos ejes principales: medios educativos e infraestructura física. Ambas condiciones hacen parte de las políticas establecidas por el MEN (Castillo, 2013) con el fin de otorgar los registros calificados a los programas académicos. Estos medios son descritos por Castillo así:

\begin{tabular}{|l|l|}
\hline $\begin{array}{l}\text { Medios } \\
\text { educativos }\end{array}$ & $\begin{array}{l}\text { Garantizar la disponibilidad de recursos bibliográficos, bases de datos, equipos y aplicativos } \\
\text { informáticos, sistemas de interconectividad, laboratorios físicos, escenarios de simulación } \\
\text { virtual y de experimentación y práctica, talleres con instrumentos acordes con la metodología } \\
\text { a presentar. } \\
\text { Para los programas a distancia y virtuales describir la disponibilidad de una plataforma } \\
\text { apropiada, la infraestructura de conectividad, las estrategias metodológicas para su } \\
\text { desarrollo, así como las estrategias de seguimiento (Castillo, 2013, p. 12). }\end{array}$ \\
\hline $\begin{array}{l}\text { Infraestructura } \\
\text { física }\end{array}$ & $\begin{array}{l}\text { La Ies [institución de educación superior] debe demostrar que la infraestructura inmobiliaria } \\
\text { para el desarrollo del programa cumple con las normas de uso del suelo de conformidad con } \\
\text { las disposiciones locales del lugar de ofrecimiento en cuya jurisdicción se desarrollará el } \\
\text { programa. En el caso de programas a distancia y virtuales la ies debe describir y demostrar } \\
\text { la disponibilidad de la infraestructura de hardware y conectividad: Software que permita } \\
\text { la producción de materiales, la disponibilidad de aulas virtuales y aplicativos para la } \\
\text { administración de procesos de formación (Castillo, 2013, p. 12). }\end{array}$ \\
\hline
\end{tabular}

Teniendo en cuenta que la pandemia movilizó abruptamente las dinámicas con las que se venían trabajando, fue necesario analizar cómo las condiciones físicas/estructurales (conectividad, espacios físicos, recursos, etc.) brindadas institucionalmente impactaron al estudiantado.

\section{Método}

La etnografía, como método de investigación cualitativa (Maturana y Garzón, 2015), permite "realizar una descripción rigurosa de lo que sucede al interior de las escuelas multiculturales y, por tanto, una mejor comprensión de los procesos educativos" (Ogbu, 1993). Los estudios etnográficos en educación autorizan una reflexión en torno a las situaciones o experiencias vividas en el contexto escolar, para contribuir a la transformación social. Maturana y Garzón (2015) describen la etnografía como un método de investigación pertinente al servicio del docente, el cual posibilita un amplio entendimiento "del contexto educativo o las particularidades del contexto escolar".

El presente estudio se enmarca en la microetnografía como parte de los estudios etnográficos en educación. Este tipo de estudio permite el análisis de una situación dada (Spradley,1980). Así, dada la contingencia sanitaria a nivel mundial, los docentes se enfrentaron al reto de documentar, observar, analizar, reflexionar sobre las metodologías emergentes, las actitudes, las percepciones, las políticas y, en general, todo lo relacionado con el ámbito escolar, para hacer frente a este nuevo desafío.

La Tabla 1 condensa la metodología, el diseño de investigación, la unidad de análisis, los instrumentos y las técnicas de investigación, y las fases implementadas para el análisis de resultados en este estudio.

Cabe resaltar que durante la ejecución de cada una de las etapas de investigación, se tuvieron en cuenta criterios éticos y bioéticos, como la confidencialidad en la información personal de los participantes, el tratamiento de los datos, los riesgos y beneficios de la investigación, a partir del uso del debido consentimiento y asentimiento informado (documentos escritos brindados por algunos directivos de la institución); la garantía 
Tabla 1 Proceso metodológico

\begin{tabular}{|c|c|}
\hline Proceso metodológico & Descripción \\
\hline Tipo y diseño de investigación & Investigación cualitativa / Diseño etnográfico: microetnografía \\
\hline Participantes & $\begin{array}{l}\text { Estudiantes activos de la Licenciatura en Lenguas Modernas con Énfasis en Inglés y Francés } \\
\text { de la Universidad del Quindío, Colombia. Se contó con la participación voluntaria de } 423 \\
\text { estudiantes activos (pertenecientes a la jornada diurna y nocturna, desde primero a décimo } \\
\text { semestre). }\end{array}$ \\
\hline Instrumentos y técnicas de investigación & $\begin{array}{l}\text { Cuestionario abierto / Entradas de texto libre (Hernández et al., 2014). Centrado en la } \\
\text { satisfacción en la experiencia de aprendizaje mediada por tecnología digital. } \\
\text { Grupos focales (Escobar y Bonilla, 2011). Participaron } 120 \text { estudiantes pertenecientes a cuatro } \\
\text { grupos de cursos básicos de Inglés, quienes ya habían respondido al cuestionario abierto. } \\
\text { Observación participativa (Spradley, 1980). Uno de los docentes investigadores como parte de la } \\
\text { metodología pat }\end{array}$ \\
\hline Análisis de resultados (fase l) & $\begin{array}{l}\text { Cuestionario abierto: se implementó un proceso de condensación, visualización e interpretación } \\
\text { de datos, que sigue a Miles et al. (2014). Este fue un análisis iterativo de dos ciclos, que se } \\
\text { realizó utilizando el software NVivo } 12 \text {. } \\
\text { Grupos focales y observaciones: también se tomaron condiciones generales en la codificación, } \\
\text { expuestas por McCracken (1988). Estas consisten en leer las transcripciones y hacer } \\
\text { anotaciones, describir categorías basadas en las transcripciones, conectar patrones y códigos, } \\
\text { agrupar temas básicos y examinar los temas }\end{array}$ \\
\hline Análisis de resultados (fase 2) & Triangulación metodológica (Okuda y Gómez, 2005). Se analizan los resultados obtenidos desde \\
\hline Triangulación & los diferentes instrumentos/técnicas/métodos para profundizar y conocer mejor el tema \\
\hline
\end{tabular}

de una conducta científica adecuada evidenciada, entre otros elementos, por el buen uso de las referencias bibliográficas, y el planteamiento de criterios FINER (acrónimo de factible, interesante, novedoso, ético y relevante) (Hulley et al., 2013) para delimitar la pregunta de investigación.

\section{Recolección de datos}

Las técnicas e instrumentos de investigación fueron aplicados en la Licenciatura de Lenguas Modernas con énfasis en Inglés y Francés de la
Universidad del Quindío, registrada en el MEN bajo la modalidad presencial, contando con 16 semanas de aprendizaje semestral. Sin embargo, debido a la situación generada por la covid-19, los estudiantes tuvieron que asistir a más de la mitad del semestre (nueve semanas) bajo la metodología PAT, y siguiendo los lineamientos para el plan de contingencia derivados del MEN.

En la Figura 2 se presenta el procedimiento para la recolección de datos.

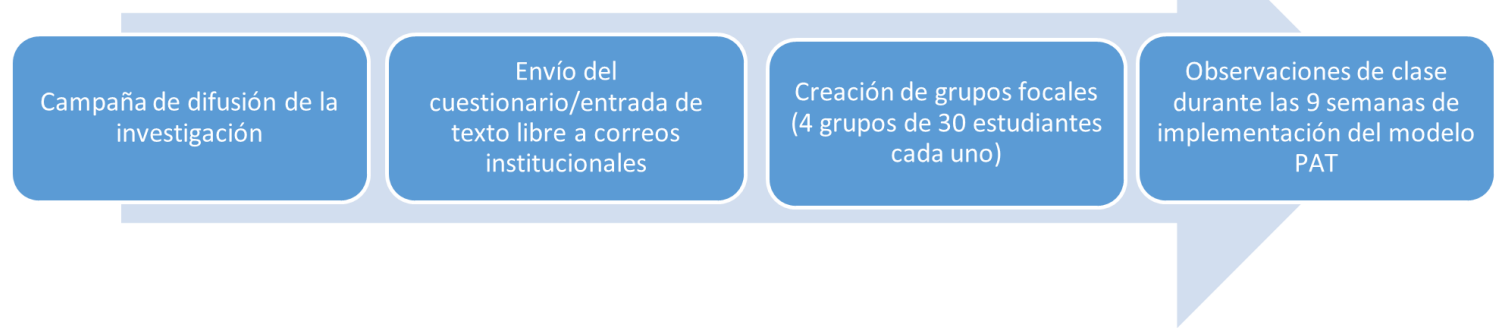

Figura 2 Procedimiento para la recolección de datos 
Al finalizar el primer semestre académico, los investigadores de este estudio realizaron una campaña de difusión de la investigación con la ayuda de los demás profesores del programa académico. Posteriormente, los estudiantes fueron contactados por medio del correo electrónico institucional, invitándolos a participar del estudio a través de un enlace que los llevaba a resolver preguntas sobre su satisfacción en la experiencia de aprendizaje mediada por tecnología digital. Igualmente, se preguntó sobre las oportunidades de mejoramiento en situaciones de confinamiento similares.

Asimismo, en el primer periodo de 2020 se organizaron cuatro grupos focales de 30 estudiantes cada uno; se indagó, especialmente, sobre aspectos psicosociales y condiciones educativas que podrían tener un impacto en la experiencia de aprendizaje.

Durante las nueve semanas con la metodología PAT, se realizaron observaciones participativas que permitieron, posteriormente, contribuir con el análisis de resultados.

\section{Resultados}

Para dar respuesta al objetivo de investigación, se identificaron aspectos psicosociales y condiciones educativas de estudiantes pertenecientes a la Licenciatura en Lenguas Modernas con énfasis en Inglés y Francés de la Universidad del Quindío que impactaron la experiencia de aprendizaje en línea bajo la estrategia metodológica PAT durante el primer periodo de confinamiento (2020-I). Es a partir de estas categorías apriorísticas que se develan los resultados.

\section{Aspectos psicosociales y su impacto en la experiencia de aprendizaje en línea}

Aunque los estudiantes que participaron en la investigación reconocen el esfuerzo y las condiciones difíciles que tuvieron que afrontar los profesores para impartir sus cursos (como se manifestó en los grupos focales), el análisis de frecuencia permitió identificar que el apoyo del profesor representa la barrera más usualmente mencionada para una experiencia satisfactoria por medio de metodología PAT. La Figura 3 muestra la frecuencia de aspectos psicosociales reportadas por los estudiantes.

Más específicamente, la ausencia de empatía, una metodología inapropiada, la falta de realimentación y comunicación, así como la falta de seguimiento por parte del profesor, constituyeron los obstáculos más recurrentes para los estudiantes. En la Tabla 2, se condensan algunas de esas opiniones de los estudiantes en las respuestas generadas a partir del cuestionario, así como sus comentarios en los grupos focales.

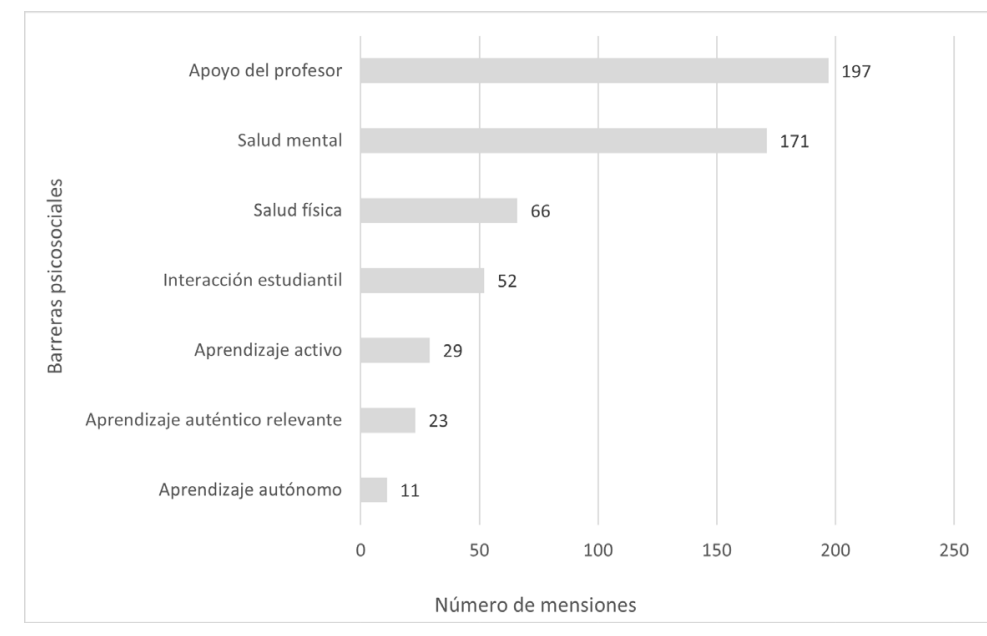

Figura 3 Aspectos psicosociales reportados por estudiantes en la aplicación de la metodología PAT 
Tabla 2 Categoría aspectos psicosociales y su impacto en la experiencia de aprendizaje en línea. Comentarios de los estudiantes en entradas de texto libre y grupos focales.

\begin{tabular}{|c|c|c|c|c|c|}
\hline Apoyo docente & $\begin{array}{l}\text { Interacción } \\
\text { estudiantil }\end{array}$ & $\begin{array}{c}\text { Aprendizaje } \\
\text { activo }\end{array}$ & $\begin{array}{l}\text { Aprendizaje } \\
\text { auténtico y } \\
\text { relevante }\end{array}$ & $\begin{array}{l}\text { Aprendizaje } \\
\text { autónomo }\end{array}$ & Salud física y mental \\
\hline $\begin{array}{l}\text { Est 38:* "La } \\
\text { cantidad de trabajos } \\
\text { incrementó y la } \\
\text { mayoría de los } \\
\text { profesores no son } \\
\text { flexibles con los } \\
\text { tiempos de entrega. } \\
\text { Todos los profes } \\
\text { utilizan plataformas } \\
\text { diferentes" } \\
\text { Est 140: "Los } \\
\text { profesores no } \\
\text { contestan rápido } \\
\text { a los correos para } \\
\text { resolver nuestras } \\
\text { dudas" } \\
\text { Est 20: "No hay } \\
\text { retroalimentación } \\
\text { después de los } \\
\text { exámenes; a veces } \\
\text { la plataforma } \\
\text { da solo la nota, } \\
\text { sin mostrar los } \\
\text { comentarios a cada } \\
\text { respuesta" }\end{array}$ & $\begin{array}{l}\text { Est 11: "La } \\
\text { interacción en línea } \\
\text { es nula, porque } \\
\text { cada quien trabaja } \\
\text { como puede y } \\
\text { por el exceso de } \\
\text { actividades no hay } \\
\text { mucho tiempo para } \\
\text { socializar" }\end{array}$ & $\begin{array}{l}\text { Est: } 328 \text { "La } \\
\text { manera actual en } \\
\text { la que se orientan } \\
\text { las clases de } \\
\text { manera digital } \\
\text { se convierte en } \\
\text { un monólogo por } \\
\text { parte del maestro } \\
\text { en muchas } \\
\text { ocasiones" }\end{array}$ & $\begin{array}{l}\text { Est 95: "Mi punto es } \\
\text { que los estudiantes } \\
\text { están pagando más } \\
\text { por que le envíen } \\
\text { tareas sin propósito } \\
\text { significativo alguno, } \\
\text { y no por una buena } \\
\text { educación" }\end{array}$ & $\begin{array}{l}\text { Est 10: "No he } \\
\text { podido organizar } \\
\text { mi tiempo por } \\
\text { la cantidad de } \\
\text { trabajo extra } \\
\text { que ponen los } \\
\text { profesores" }\end{array}$ & $\begin{array}{l}\text { Est 69: "[...] dejan más } \\
\text { trabajos de los normales, } \\
\text { creando así más estrés del } \\
\text { que ya tenemos; además, } \\
\text { nunca están presentes si } \\
\text { tenemos alguna dificultad" }\end{array}$ \\
\hline
\end{tabular}

* El número correspondiente a cada estudiante se asigna teniendo en cuenta la matriz obtenida para el análisis de información con el software NVivo.

[ ] información adicional para un mejor entendimiento de la oración.

Se evidencian claramente algunas variables que intervienen en las percepciones de los estudiantes en cuanto a los aspectos psicosociales que influyen en el aprendizaje en línea. Estas percepciones van en dos direcciones: por un lado, el docente, quien aplica una metodología, asigna unas tareas y regula de alguna manera la interacción. Por otro lado, el estudiante, con factores individuales (como la organización del tiempo de trabajo), es quien ve cómo muchos de sus procesos de aprendizaje se ven afectados.
Cabe resaltar que la interacción resulta ser uno de los procesos del aprendizaje más impactados, bien sea con sus compañeros (la interacción en línea es nula) o con sus profesores (realimentación de trabajos, tiempo para responder correos, etc.).

Estos hallazgos permiten reflexionar sobre la necesidad de una aplicación correcta de los lineamientos planteados para la educación virtual y a distancia, enfatizando en la importancia de la capacitación docente para afrontar los cambios que las nuevas dinámicas educativas implican. 


\section{Condiciones educativas}

El análisis de las respuestas de los estudiantes obtenidas en el diálogo en los grupos focales permitió constatar que las condiciones educativas (medios educativos) tienen un impacto importante en todos los componentes psicosociales, incluido el apoyo del profesor. Algunas opiniones que ilustran estas dificultades se pueden contemplar en la Tabla 3.

Los resultados obtenidos desde las respuestas de entrada libre y los grupos focales fueron constatados desde la observación participante, que permitió evidenciar cómo la falta de conectividad o inestabilidad en la conexión, el espacio inadecuado y la falta de dispositivos electrónicos hacen que las percepciones de los estudiantes revelen insatisfacción en múltiples aspectos psicosociales. Dichos hallazgos van en dirección con lo evidenciado desde otras investigaciones (Arias et al., 2020; Chicaiza, 2020; Lovón y Cisneros, 2020; Ramírez et al., 2020; Valdieso et al., 2020), resaltando la importancia de contar con condiciones

Tabla 3 Comentarios de los estudiantes acerca de la relación de las condiciones educativas con los aspectos psicosociales

\begin{tabular}{|c|c|c|c|c|c|}
\hline $\begin{array}{c}\text { Sub } 1 \\
\text { Apoyo docente }\end{array}$ & $\begin{array}{c}\text { Sub } 2 \\
\text { Interacción } \\
\text { estudiantil }\end{array}$ & $\begin{array}{c}\text { Sub } 3 \\
\text { Aprendizaje } \\
\text { activo }\end{array}$ & $\begin{array}{c}\text { Sub } 4 \\
\text { Aprendizaje } \\
\text { auténtico y } \\
\text { relevante }\end{array}$ & $\begin{array}{c}\text { Sub } 5 \\
\text { Aprendizaje } \\
\text { autónomo }\end{array}$ & $\begin{array}{c}\text { Sub. } 6 \\
\text { Salud física y } \\
\text { mental }\end{array}$ \\
\hline $\begin{array}{l}(05: 10) \\
\text { "Presencialmente, } \\
\text { el aprendizaje } \\
\text { es mejor y no } \\
\text { presenta fallas, } \\
\text { mientras que } \\
\text { virtualmente } \\
\text { los profesores } \\
\text { enfrentan } \\
\text { demasiadas fallas } \\
\text { técnicas en sus } \\
\text { dispositivos } 0 \\
\text { plataformas" }\end{array}$ & $\begin{array}{l}\text { (08:00) "Muchas veces no } \\
\text { pude participar por falta } \\
\text { de internet" } \\
\text { (08:30) "La interacción } \\
\text { con tus compañeros } \\
\text { depende muchísimo } \\
\text { de tu capacidad para } \\
\text { conectarte a internet. Así } \\
\text { como hay compañeros } \\
\text { que se apoyan entre sí } \\
\text { para adaptarse mejor } \\
\text { a las clases virtuales, } \\
\text { hay otros a los que } \\
\text { simplemente les cuesta } \\
\text { comunicarse" } \\
\text { (11:40) "Hay profesores } \\
\text { que utilizan plataformas } \\
\text { donde se pueden formar } \\
\text { grupos, pero cada que lo } \\
\text { hacen no puedo continuar } \\
\text { con la actividad, porque } \\
\text { la plataforma me expulsa } \\
\text { inmediatamente" }\end{array}$ & $\begin{array}{l}\text { (07:15) "Realmente } \\
\text { considero que } \\
\text { mi aprendizaje } \\
\text { activo fue casi } \\
\text { nulo, no cuento con } \\
\text { conexión a internet } \\
\text { cerca de mi casa y } \\
\text { tampoco con medios } \\
\text { tecnológicos, por } \\
\text { lo que ha sido } \\
\text { una experiencia } \\
\text { bastante difícil y me } \\
\text { siento excluida" }\end{array}$ & $\begin{array}{l}\text { (15:45) "Los } \\
\text { estudiantes no } \\
\text { cuentan con la } \\
\text { tecnología e } \\
\text { internet de buena } \\
\text { calidad para } \\
\text { trabajar estas } \\
\text { actividades. Con } \\
\text { mucho esfuerzo y } \\
\text { dedicación, trabajo } \\
\text { y estudio con mi } \\
\text { celular, pero no } \\
\text { es lo ideal. En mi } \\
\text { opinión, es mejor } \\
\text { poco trabajo de } \\
\text { buena calidad que } \\
\text { mucho trabajo de } \\
\text { mala calidad" }\end{array}$ & $\begin{array}{l}\text { (12:00) "Es } \\
\text { muy difícil } \\
\text { concentrarse, } \\
\text { debido a que } \\
\text { en los ámbitos } \\
\text { familiares no } \\
\text { hay espacios } \\
\text { ni ambientes } \\
\text { apropiados para } \\
\text { el aprendizaje. } \\
\text { Hay demasiados } \\
\text { estímulos } \\
\text { alrededor de } \\
\text { los estudiantes } \\
\text { que dificultan } \\
\text { las variables de } \\
\text { atención en los } \\
\text { estudiantes" }\end{array}$ & $\begin{array}{l}\text { (25:15) "Tuve } \\
\text { que soportar un } \\
\text { desequilibrio } \\
\text { emocional muy } \\
\text { fuerte, pasar a ser } \\
\text { la única fuente } \\
\text { de ingresos en } \\
\text { la casa y aparte } \\
\text { la exigencia para } \\
\text { intentar continuar } \\
\text { normalmente el } \\
\text { aprendizaje (cosa } \\
\text { que no logré). } \\
\text { Resulta muy } \\
\text { desgastante". } \\
\text { (31:00) "Es muy } \\
\text { estresante estar } \\
\text { todo el día al frente } \\
\text { de un computador, } \\
\text { termino más } \\
\text { cansada, muchas } \\
\text { veces muy } \\
\text { frustrada por } \\
\text { problemas técnicos } \\
\text { y de conexión" }\end{array}$ \\
\hline
\end{tabular}

Convenciones en la trascripción de datos:

(00:00) tiempo en que se mencionó el comentario.

Cursiva: oraciones en el discurso que resaltan las condiciones educativas. 
educativas óptimas para no afectar el proceso de enseñanza-aprendizaje y todo lo que ello implica.

La Figura 4 resume cómo, en la identificación de factores, el apoyo del profesor y las condiciones educativas son los aspectos de mayor relevancia, ya que estos tienen una incidencia tanto directa como indirecta en los componentes psicosociales y en la satisfacción estudiantil.

La identificación de factores que se exponen en este estudio indica que las condiciones educativas son la piedra angular en la experiencia de aprendizaje con la metodología PAT. En este sentido, existen estudios que ya apuntaban la dicotomía inclusión-inclusión digital presente en el contexto colombiano (Rueda y Franco-Avellaneda, 2018). Si bien hay planteamientos que señalan que no se puede alcanzar la justicia social sin conectividad digital, y que esta debe ser fundamental en Colombia (López y Gómez, 2020; Restrepo y Gómez, 2020), lo cierto es que la pandemia es un evento disruptivo que afectó la puesta en marcha de políticas educativas pensadas para este fin.

\section{Discusión}

Los resultados del presente estudio resaltan los aspectos psicosociales y las condiciones educativas también halladas en otros contextos y latitudes; como lo evidenciado en Arias et al., 2020, quienes encuentran que un alto porcentaje de estudiantes universitarios $(46,8 \%)$ han notado cambios en sus estados de humor durante la pandemia, lo que ha afectado procesos académicos y la interacción entre pares; o en Chandra (2020), cuya investigación establece que los estudiantes experimentan estrés debido a situaciones relacionadas con aspectos psicoculturales y psicológicos por el contexto de pandemia transversal a todos los lugares del mundo; o en Ramírez et al. (2020), quienes concluyen su investigación afirmando que las percepciones de los estudiantes en cuanto a la educación virtual no son buenas, destacando, entre otras razones, factores socioeconómicos.

El apoyo docente, como una de las barreras psicosociales de mayor recurrencia en las entradas de texto libre y como un punto clave de discusión en los grupos focales, es sin duda uno de los criterios que necesitan mayor atención. $Y$ es que, como lo afirman McNatt et al., "un apoyo psicosocial adecuado y bien diseñado puede mejorar los procesos resilientes y mitigar las vulnerabilidades" (2018, p. 9). En este sentido, la educación, y particularmente el apoyo del profesor, puede constituirse en el canal principal para reducir la brecha entre algunas barreras psicosociales y el aprendizaje, como la propuesta por la Red Interinstitucional para la Educación en Situaciones de Emergencia (McNatt et al., 2018) que plantea una "nota de

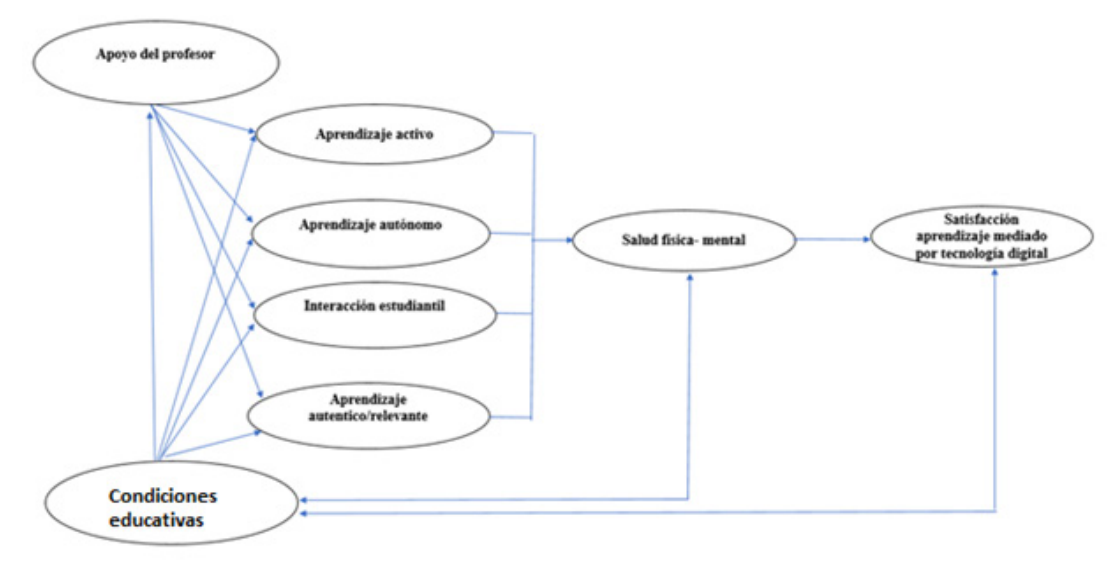

Figura 4 Identificación de factores que influyen en la satisfacción del aprendizaje en el contexto PAT 
orientación" para dicho apoyo psicosocial en el contexto educativo.

En el campo de las lenguas extranjeras, Díaz y Jansson (2011) concluyen que el rol del profesor es muy diverso y que este "varía dependiendo de las tareas a enseñar con la ayuda de la tecnología”. En tal sentido, el profesor puede desempeñar el rol de "controlador", ya que selecciona cuáles son las estrategias que mejor funcionen; "organizador" de todo el proceso, tanto de enseñanza como de aprendizaje; "asesor", al participar con los estudiantes en las tareas realizando retroalimentación, y "diseñador" de estrategias innovadoras que ayuden al logro de objetivos. Es decir, la educación virtual requiere de una diversidad de los roles del docente en la educación.

Así, es deber de los docentes repensar cuál debería ser su rol cuando se enfrente a una metodología como el pat. Ya lo advertía Vega en el año 1999, en su texto "El docente del siglo XXI: formación y retos pedagógicos": el uso de las tecnologías de la información y la comunicación se convierte en un instrumento sociocultural que debe incorporarse a los procesos de enseñanza aprendizaje con prontitud y los docentes deben estar capacitados para afrontar el acelerado ritmo con que va creciendo el uso y la importancia de las Tic.

Observaciones de los estudiantes respecto a factores metodológicos como la falta de empatía, la falta de flexibilidad, la monotonía y la falta de creatividad demuestran la necesidad de reflexionar en algunas estrategias que se deben tener en cuenta al momento de afrontar la metodología PAT. Y es que los docentes se vieron enfrentados a un reto para el cual no estaban capacitados. Como lo discutía Mirete Ruiz (2010) en su reflexión sobre la formación docente en el uso de las TIC, los estudiantes son nativos digitales y los docentes deben estar preparados a los cambios que impone la sociedad; en este sentido, deben estar preparados para la inclusión de las TIC en el aula como eje facilitador del aprendizaje, y para esto, se requiere de una formación pedagógica y de unas "competencias instrumentales para usar el software y hardware"(p. 42) que permitan una inserción asertiva de la tecnología en las aulas.

Es por esta razón por lo que, además de las medidas que aseguren una democratización en el acceso a tecnología digital, se debe hacer hincapié en la formación profesoral en metodología PAT. Desde un punto de vista de políticas educativas, el estudio sugiere la articulación de un grupo de expertos en educación mediada por tecnología digital, el cual produzca lineamientos generales que sirvan como punto inicial para el desarrollo de capacitaciones a los docentes en enseñanza durante metodología PAT.

Por su parte, entre los desafíos que enfrentan los estudiantes de lenguas extranjeras frente a este nuevo modelo, se encuentra trascender en niveles ulteriores de autonomía; es decir, pasar de un nivel de autonomía aproximación-implicación, en el cual se reconocen las fortalezas y debilidades a nivel lingüístico-didáctico, a niveles superiores de autonomía, como el nivel de investigación-intervención y el nivel lingüístico didáctico, los cuales exigen, a parte de un reconocimiento de los estilos de aprendizaje y la selección de estrategias para afrontar el aprendizaje de lenguas, una capacidad de reflexión y proyección de lo aprendido hacia la resolución de problemas (Velázquez et al., 2016).

Respecto a las condiciones educativas, es evidente que no había una preparación a priori para enfrentar la situación disruptiva que cambió todas las dinámicas que se venían implementando desde la metodología presencial; los estudiantes no contaban con las condiciones básicas para trasladar la presencialidad a la virtualidad; algunos no tenían los dispositivos necesarios, conexión a internet estable, o un espacio adecuado para el aprendizaje, y hubo factores socioeconómicos que impidieron un óptimo traslado de modalidad. De allí, entonces, la importancia de enfatizar en el uso de las tecnologías móviles, aún cuando sea factible regresar a las aulas. 
Si bien los estudiantes podrían ser llamados "nativos digitales", es su mayor reto canalizar y direccionar todos sus conocimientos digitales hacia procesos de formación que ayuden a mejorar su desempeño en lenguas extranjeras.

Es menester resaltar que el Gobierno debe implementar políticas educativas ya plasmadas en papel, como el Plan Nacional de Desarrollo 2018-2022 (Colombia, Congreso de la República, 2019) y el CONPES 3988 de 2020 en lo que respecta a medidas como la modernización de las telecomunicaciones, la implementación de programas de acceso y servicio universal de las TIC en todo el territorio nacional, la masificación del acceso a internet, el apoyo a la educación pública con conectividad, la formación a los usuarios para el uso de las tecnologías, y el subsidio para incentivar la conectividad en familias vulnerables; todo esto con el fin de mantener un sistema de educación PAT exitoso. Sobre todo, teniendo en cuenta que la educación tiene el riesgo de enfrentar una creciente deserción, a causa del mayor desempleo y el importante número de personas que pasaron a formar parte del segmento de personas en pobreza absoluta (Alarcón, 2020).

Desde este estudio, y considerando que la metodología PAT podría integrarse a la modalidad "educación a distancia" como una posibilidad para perfiles de estudiantes diversos que se encuentren en diferentes latitudes, se podría presentar una propuesta macrocurricular para los programas de pregrado que deseen continuar con la implementación de este modelo. Dicha propuesta debería incluir:

1. La organización de los espacios sincrónicos y asincrónicos, dependiendo del número de horas, tipo (teórico-práctico) y créditos del espacio académico.

2. La parametrización de contenidos en una única plataforma institucional que congregue a todos los estudiantes.

3. La creación de lineamientos concretos para cada encuentro. En los encuentros sincrónicos, la posibilidad de grabar las sesiones (bajo consentimiento de cada uno de los estudiantes) para compartirlas posteriormente (garantizando flexibilidad y empatía hacia los problemas técnicos que puedan surgir); en los asincrónicos, procurar la organización del contenido de modo tal que se consideren todos los estilos de aprendizaje.

4. El aseguramiento de la interacción entre los participantes.

5. La capacitación docente como eje central para garantizar la satisfacción del aprendizaje en línea.

Ha transcurrido un año a partir del uso de la metodología PAT a causa de la pandemia; las medidas que ahora se vienen planteando desde el Gobierno nacional y que son analizadas y reestructuradas desde los Gobiernos locales y desde las instituciones, incluyen el retorno progresivo a clase a partir de la "alternancia educativa" y la metodología "clase espejo"; es así como, para futuras investigaciones, se espera indagar sobre las percepciones de la comunidad académica respecto a estas estrategias y continuar en la búsqueda del mejor escenario para sobrellevar situaciones como la que hoy por hoy nos aqueja.

\section{Conclusión}

El presente estudio se interesó por identificar los aspectos psicosociales y las condiciones educativas de estudiantes de Licenciatura en Lenguas Modernas con énfasis en inglés y francés de la Universidad del Quindío que impactan su experiencia de aprendizaje durante la implementación de la metodología PAT a causa del periodo de confinamiento ocasionado por el virus SARS-CoV-2 que ocasiona la enfermedad covid-19.

Por medio de métodos propios de un análisis cualitativo, microetnográfico, como grupos focales, observación participativa y encuestas con entrada libre de texto, se pudo determinar una interconexión entre diversos aspectos psicosociales $\mathrm{y}$ condiciones educativas. 
Así, el estudio sugiere que para una experiencia de aprendizaje PAT satisfactoria, se enfatice sobre dos ejes fundamentales: las condiciones estructurales que faciliten el aprendizaje (como, por ejemplo, el acceso a dispositivos digitales para el aprendizaje, una conexión de apropiada a internet, un espacio de estudio apropiado) y el apoyo del profesor al estudiante (en lo que tiene que ver con la empatía, la metodología, la realimentación, la comunicación y el seguimiento). Dichos factores cumplen un papel ineludible en otros aspectos psicosociales del estudiante, como su aprendizaje activo, aprendizaje autónomo y relevante, interacción, salud física y mental. Debido a esto, el estudio sugiere algunas acciones que pueden contribuir a enfrentar de una mejor manera las barreras en el aprendizaje por medio de la metodología PAT.

Desde la perspectiva de políticas educativas, se requiere la puesta en marcha inmediata de lineamientos decretados antes de la pandemia y que apuntan a la democratización de la tecnología digital y a la creación de un grupo de docentes especialistas que elaboren un plan de acción metodológico que aproveche los beneficios de la tecnología, utilizandola como un soporte crucial en el proceso formativo.

Desde una perspectiva metodológica, se sugiere establecer un balance entre la carga académica sincrónica y asincrónica, tomando en cuenta la opinión de los estudiantes; evitar actividades académicas extra que no contribuyan significativamente al trabajo independiente exigido en los créditos académicos y en las que solo se espera que los estudiantes sean receptores pasivos de información; utilizar una plataforma común que facilite interacción con otras herramientas tecnológicas para el aprendizaje; incentivar la creatividad y el aprendizaje activo en las actividades asincrónicas; fomentar la flexibilidad durante las clases sincrónicas, por ejemplo, permitiendo que se graben dichas clases para que los estudiantes puedan acceder a los contenidos cuando más les convenga. La implementación de dichas medidas contribuirá al grado de satisfacción de los estudiantes hacia un aprendizaje por medio de la metodología PAT.

No cabe duda de que esta metodología seguirá cumpliendo un papel importante en el contexto de aprendizaje en línea durante periodos excepcionales, como el aprendizaje durante una pandemia. De allí que continuar en la búsqueda de planes de mejoramiento que optimicen su uso es y seguirá siendo tarea imperante para docentes e investigadores.

Finalmente, cabe resaltar que este estudio contó con algunas limitantes, como la exclusión de muchos participantes del estudio, puesto que al analizar los cuestionarios algunos no contaban con toda la información requerida. De igual manera, el hecho de omitir algunas preguntas que hubiesen sido de gran interés para acercarnos a una reflexión respecto a la percepción de los estudiantes, futuros docentes, acerca de los roles que tendrán que desempeñar cuando sean maestros en condiciones como las analizadas en esta investigación.

\section{Referencias}

Alarcón, R. D. (2020). La educación digital en Colombia en tiempos de COVID 19 y su impacto en las organizaciones educativas [Ensayo para título de especialización, Universidad Militar Nueva Granada]. https:// repository.unimilitar.edu.co/bitstream/handle/10654/36658/Ensayo\%20Rub\%C3\%A9n\%20 D a r \% C 3\% A D o \% 20 Ala r c \% C 3\% B 3 n \% 20 $\mathrm{Su} \% \mathrm{C} 3 \% \mathrm{~A} 1$ rez.pdf ?sequence $=1 \&$ isAllowed $=\mathrm{y}$

Aponte, C. (2018). Clase Espejo. Caja de herramientas para la internacionalización del currículo. Herramienta N. ${ }^{\circ}$. ITM Institución Universitaria, Dirección de Cooperación y Relaciones Internacionales

Arias, B., Castañeda, A., Pecero, J. y Frías M. (2020). Percepción emocional del estudiante universitario ante el distanciamiento social aplicado por la pandemia COVID-19. Ocronos, 3(8), 52. https://revistamedica. $\mathrm{com} /$ percepcion-emocional-estudiante-universitario-distanciamiento-social-covid-19/

Asociación Colombiana de Instituciones de Educación Superior con Programas a Distancia (ACESAD). (2013). Lineamientos para la autoevaluación con 
fines de acreditación de programas de educación superior a distancia. https://www.mineducacion.gov. co/1759/articles-341892_archivo_pdf.pdf

Cabrales, A., Graham, A., Sahlberg, P., Hodges, C. H., Moore, S., Lockee, B., Trust, T., Bond, A., Lederman, D., Greene, J., Maggioncalda, J., Soares, L., Veletsianos, G. y Zimmerman. J. (2020). Enseñanza de emergencia a distancia: textos para la discusión. The Learning Factor.

Castillo, M. (2013). Lineamientos de calidad para la verificación de las condiciones de calidad de los programas virtuales y a distancia. Ministerio de Educación $\mathrm{Na}$ cional, Secretaría Ejecutiva del Convenio Andrés Bello. https://www.mineducacion.gov.co/1759/ articles-338171_archivo_pdf.pdf

Colombia, Congreso de la República de Colombia. (2019). Plan Nacional de Desarrollo 2018-2022. Bogotá.

Colombia, Departamento Nacional de Planeación (2020). Documento CONPES 3988. Tecnologias para aprender: politica nacional para impulsar la innovación en las prácticas educativas a través de las tecnologias digitales. https://colaboracion.dnp.gov.co/CDT/ Conpes/Econ\%C3\%B3micos/3988.pdf

Colombia, Ministerio de Educación Nacional (MEN) (2020c). Lineamientos para la prestación del servicio de educación en casa y en presencialidad bajo el esquema de alternancia y la implementación de prácticas de bioseguridad en la comunidad educativa. Junio 2020. https://www.mineducacion.gov. co/1759/articles-399094_recurso_1.pdf

Colombia, Ministerio de Educación Nacional (MEN). (2010). Lineamientos para la educación virtual en la educación superior. MEN. https://aprende.colombiaaprende. edu.co/ckfinder/userfiles/files/Lineamientos_para_ la_educacion_Virtual_dic_29.pdf

Chandra, Y. (2020). Online education during CoVID-19: Perception of academic stress and emotional intelligence coping strategies among college students. Asian Education and Development Studies, 10(2), 229-238. https://doi.org/10.1108/ AEDS-05-2020-0097

Chaves, A. (2017). La educación a distancia como respuesta a las necesidades educativas del siglo XXI. Academia y Virtualidad, 10(1), 23-41. https://doi. org/10.18359/ravi.2241

Chicaiza, A. (2020). Percepción sobre la educación virtual, en adolescentes de la parroquia Salasaca, durante la pandemia del covid-19. Ocronos, 3(8), 160. https://revistamedica.com/educacion-virtual-adolescentes-pandemia-COVID-19/
Díaz, C. H. y Jansson, L. (2011). El aprendizaje del inglés y el uso de tecnologías: percepciones de estudiantes y profesores de inglés del nivel secundario chileno. Matices en Lenguas Extranjeras, (5), 1-37. https:// revistas.unal.edu.co/index.php/male/article/ view/44697

Escobar, J. y Bonilla-Jimenez, F. (2011). Grupos focales: una guía conceptual y metodológica. Cuadernos Hispanoamericanos de Psicología, 9(1), 51-67. http:// www.tutoria.unam. $\mathrm{mx} /$ sitetutoria/ayuda/gfocal-03122015.pdf

Fonseca, M. (2005). El componente afectivo en el aprendizaje de lenguas. En M. Navarro et al. (Eds.), Nuevas tendencias en lingüistica aplicada (pp. 5579). Servicio Publicaciones UCAM. https://www. researchgate.net/publication/309583526_El_componente_afectivo_en_el_aprendizaje_de_lenguas

Govea Piña, L. y Sánchez, F. (2006). La lengua extranjera y la interacción verbal en el aula. Laurus, 12(22), 208-223. https://www.redalyc.org/articulo. oa? id=76102211

Hernández-Sampieri, R., Fernández, C. y Baptista, P. (2014). Selección de la muestra. En Metodología de la investigación (6. ${ }^{\text {ed.) }}$ (pp. 394-402) McGraw-Hill.

Hulley, S. B., Cummings, S. R., Browner, W. S., Grady, D. G. y Newman, T. B. (2013). Designing clinical research (4. ${ }^{a}$ ed.). Lippincott Williams and Wilkins.

Kukulska-Hulme, A. (2009). Will mobile learning change language learning? ReCALL, 21(2), 157-165. https://doi.org/10.1017/S0958344009000202

Lamí Rodríguez del Rey, L. E., Pérez Fleites, M. G. y Rodríguez del Rey Rodríguez, M. E. (2016). Las herramientas de comunicación sincrónica y asincrónica en la clase presencial. Conrado, 12(56), 84-89. https://conrado.ucf.edu.cu/index.php/conrado/ article/view/408

Leal, F. (2005). Lo psicosocial en contextos educativos: consideraciones conceptuales y empíricas a partir de una experiencia en liceos de alta vulnerabilidad. Limite, 1(12), 51-104. https://www.redalyc.org/ pdf/836/83601203.pdf

López, G. A. y Gómez, C. F. (2020). Estado de excepción y restricción al derecho a la educación en Colombia por la Covid-19. Opinión Jurídica, 19(40), 163186. https://doi.org/10.22395/ojum.v19n40a8

Lovón, M. y Cisneros, S. (2020). Repercusiones de las clases virtuales en los estudiantes universitarios en el contexto de la cuarentena por Covid 19: el caso de la PUCP. Propósitos y Representaciones. Revista de 
Psicología Educativa, 8(SPE3), e588. https:// doi. org/10.20511/pyr2020.v8nSPE3.588

Mac Callum, K., Jeffrey, L. y Kinshuk, K. (2014). Factors impacting teachers' adoption of mobile learning. Journal of Information Technology Education, 13, 141-162. https://doi.org/10.28945/1970

Maturana, G. y Garzón, C. (2015). La etnografía en el ámbito educativo: una alternativa metodológica de investigación al servicio docente. Revista Educación y Desarrollo Social, 9(2), 192-205. https://doi. org/10.18359/reds.954

McCracken, G. (1988). The long interview. Sage Publication. https://doi.org/10.4135/9781412986229

McNatt, Z, Boothby, N.,Wessells, M. y Lo, R. (2018). Nota de orientación en la INEE sobre apoyo psicosocial: facilitando el bienestar psicosocial y la psicoeducación. INEE.

Miles, M. B., Huberman, M. A. y Saldaña, J. (2014). qualitative data analysis. A methods sourcebook. Sage Publication.

Mirete Ruiz, A. B. (2010). Formación docente en TICS. ¿Están los docentes preparados para la (r)evolución TIC? International Journal of Developmental and Educational Psychology, 4(1), 35-44. https://www. redalyc.org/articulo.oa?id=349832327003

Morales, P. (2014). Metodología de educación a distancia. Universidad Santo Tomás.

Ogbu, J. (1993). Etnografía escolar. Una aproximación a nivel múltiple. En H. Velasco, F. García y A. Díaz de Rada (Eds.), Lecturas de antropología para educadores. El ámbito de la antropología de la educación y la etnografía escolar (pp. 145-174). Trotta. https:// www.researchgate.net/publication/259673956 Lecturas_de_antropologia_para_educadores_El_ ambito_de_la_antropologia_de_la_educacion_y_ la_etnografia_escolar

Okuda, M. y Gómez, C. (2005). Métodos en investigación cualitativa: triangulación. Revista Colombiana de Psiquiatria, 34(1), 118-124. https://www.redalyc. org/pdf/806/80628403009.pdf

Pardo, C. (2020). Coronavirus y el futuro de la educación de lenguas extranjeras a nivel universitario. World Languages and Cultures Publications, 231. https:// lib.dr.iastate.edu/language_pubs/231

Pérez, M. (2009). La comunicación y la interacción en contextos virtuales de aprendizaje. Apertura, 1(1), 1-21. http://www.udgvirtual.udg.mx/apertura/index. $\mathrm{php/apertura/article/view/15/18}$
Ramírez, I., Jaliri, C., Méndez, B. y Orlandini, I. (2020). Percepciones universitarias sobre la educación virtual. Red de docentes IB, 3(1), 1-6. https://www.aacademica.org/ivonne.fabiana.ramirez.martnez/11.pdf

Redacción Educación El Tiempo. (2020, 8 de septiembre). ¿En qué va el regreso a clases presenciales en las universidades? El Tiempo. https://www.eltiempo.com/ vida/educacion/regreso-a-clase-en-que-va-el-retorno-a-las-aulas-en-las-universidades-536550

Restrepo, J. F.y Gómez, D. S. (2020). La conectividad digital como derecho fundamental en colombia. Revista de Direito, Estado e Telecomunicaçôes, 12(1), 113-136. https://doi.org/10.26512/lstr.v12i1.31161

Romero, J., García, M., Roca, C., Sanjuán A. y Pulido, A. (2014). Diseño de un aprendizaje adaptado a las necesidades del alumno. Teoría de la Educación. Educación y Cultura en la Sociedad de la Información, 15(3), 172-189. https://www.redalyc.org/ pdf/2010/201032662010.pdf

Rueda, R. y Franco-Avellaneda, M. (2018). Políticas educativas de TIC en Colombia: entre la inclusión digital y formas de resistencia-transformación social. Pedagogia y saberes, (48), 9-25. https://doi.org/10.17227/ pys.num $48-7370$

Spradley, J. P. (1980). Participant observation. Rinehart \& Winston.

Tünnermann Bernheim, C. (2011). El constructivismo y el aprendizaje de los estudiantes. Universidades, (48), 21-32. https://www.redalyc.org/articulo. oa? id=37319199005

Universidad EAN (2020, 13 de marzo). Comunicado institucional 2. Gracias a la tecnología, la Universidad EAN continuará con sus actividades académicas durante el plan de contingencia por COVID-19. https:// universidadean.edu.co/sites/default/files/ institucion/comunicados/Comunicado-institucional-Covid19-2.pdf

Valdivieso, M., Burbano, V. y Burbano, A. (2020). Percepción de estudiantes universitarios colombianos sobre el efecto del confinamiento por el coronavirus, y su rendimiento académico. Revista Espacios, 41(42), 269-281. https://doi.org/10.48082/ espacios-a20v41n $42 \mathrm{p} 23$

Vega, L. (1999). El docente del siglo XXI: formación y retos pedagógicos. Revista Española de Educación Comparada, (5), 209-230. http://revistas.uned.es/index. $\mathrm{php} / \mathrm{REEC} /$ article/view/7274/6942

Velázquez, Y., Pérez, A. y Parra, J. (2016). Los niveles de autonomía en el aprendizaje del profesor en formación 
de lenguas extranjeras.Didasc@lia:DidácticayEducación, 7(1), 85-96 [Acceso restringido].

Walker, S. L. y Fraser, B. J. (2005). Development and validation of an instrument for assessing distance education learning environments in higher education: The Distance Education Learning Environments Survey (DELES).Learning Environ Res, 8, 289-308. https:// doi.org/10.1007/s10984-005-1568-3

Cómo citar este artículo: García Botero, J., García Botero, G. y Botero Restrepo, M. A. (2021). Aspectos psicosociales y condiciones educativas de docentes de lengua en formación durante el confinamiento por la pandemia de Covid-19. Íkala, Revista de Lenguaje y Cultura, 26(3), 553-569. https://doi. org/10.17533/udea.ikala.v26n3a05 
